\title{
An adaptive model for gas-liquid mass transfer in a Taylor vortex reactor
}

\author{
Xi Gao ${ }^{\mathrm{a}}$, Bo Kong ${ }^{\mathrm{a}}$, Mahdi Ramezani ${ }^{\mathrm{b}}$, Michael G. Olsen ${ }^{\mathrm{b}}$, R. Dennis Vigila,* \\ ${ }^{a}$ Department of Chemical \&s Biological Engineering, Iowa State University, Ames, IA 50011 \\ ${ }^{b}$ Department of Mechanical Engineering, Iowa State University, Ames, IA 50011
}

\begin{abstract}
Gas-liquid Taylor-Couette flow devices have attracted interest for use as chemical and biological reactors, and consequently the accurate prediction of interphase mass transfer coefficients is crucial for their design and optimization. However, gas-liquid mass transport in these systems depends on many factors such as the local velocity field, turbulent energy dissipation rate, and the spatial distribution and size of bubbles, which in turn have complicated dependencies on process, geometric, and hydrodynamic parameters. Here we overcome these problems by employing a recently developed and validated Eulerian two-phase CFD model to compute local values of the mass transfer coefficient based upon the Higbie theory. This approach requires good estimates for mass transfer exposure times, and these are obtained by using a novel approach that automatically selects the appropriate expression (either the penetration model or eddy cell model) based upon local flow conditions. By comparing the simulation predictions with data from corresponding oxygen mass transfer experiments, it is demonstrated that this adaptive mass transfer model provides an excellent description for both the local and global mass transfer of oxygen in a semibatch gas-liquid Taylor-Couette reactor for a wide range of azimuthal Reynolds numbers and axial gas flow rates.
\end{abstract}

Keywords: Mass Transfer, Taylor-Couette Flow, Gas-Liquid Flow, CFD

\footnotetext{
${ }^{*}$ Corresponding author

Email address: vigil@iastate.edu (R. Dennis Vigil)
}

Preprint submitted to International Journal of Heat and Mass Transfer

July 28, 2015

(C) 2015. This manuscript version is made available under the Elsevier user license http://www.elsevier.com/open-access/userlicense/1.0/ 
Simulation

\section{Introduction}

Interphase mass transfer plays a crucial role in the design, scale-up and optimization of multiphase chemical and biological reactors. As a result, considerable effort has been expended to develop reliable correlations for estimating interphase mass transfer coefficients. For gas-liquid systems, it is usually assumed that the liquid side mass transfer resistance at gas-liquid interfaces limits interphase mass transport, and therefore gas side mass transfer resistance is neglected [1]. Hence, the liquid side volumetric mass transfer coefficient $\left(k_{L} a\right)$ is used to compute the overall mass transfer rate across a gas-liquid interface. However, it can be difficult to estimate $k_{L} a$ because of the many factors affecting this quantity, such as gas holdup and bubble size, slip velocity, and turbulent energy dissipation rate. These factors in turn depend non-trivially on reactor operating conditions, geometry, and physical properties of the gas and liquid phases. Some dependencies of the volumetric mass transfer coefficient on hydrodynamic, operating, and geometric parameters are illustrated in Fig. 1.

Although numerous empirical correlations have been developed for gas-liquid mass transfer in bubble columns $[2,3,4,5]$, airlift reactors $[6,7,8]$, and stirred tanks $[9,10,11,12]$, comparatively little is known concerning interphase mass transfer in Taylor-Couette flow cells $[13,14,15,16]$, which have recently gained interest for use as bioreactors $[17,18,19,20,21,22,23,24]$. These devices, which consist of fluids confined in the annular space between two coaxial cylinders (see Fig. 2) with the inner cylinder undergoing rotation, can be used to generate pairs of toroidal vortices with mixing characteristics advantageous for culturing a variety of microorganisms $[17,18,19,21,22]$. Specifically, as the inner cylinder rotation speed increases above a critical value that depends upon the reactor geometry and fluid properties, the fluid undergoes transition from laminar Couette flow (circular flow with only an azimuthal component) to laminar Taylor vortex flow. Subsequent increases in cylinder rotation speed lead 


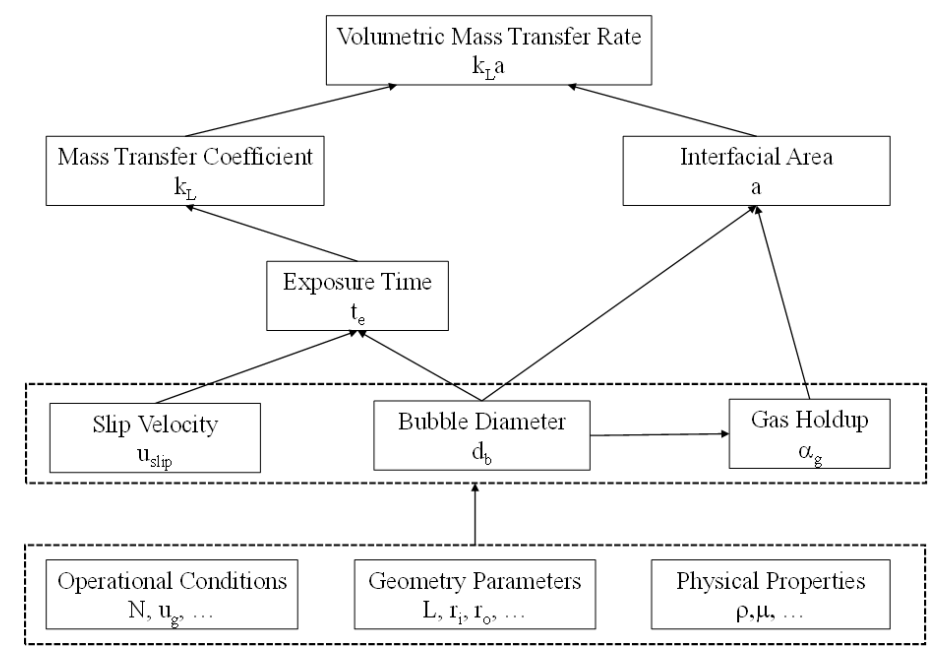

Figure 1: Illustration of the relationships between volumetric mass transfer coefficient and various geometric, operational, and hydrodynamic parameters.

to higher order instabilities such as wavy vortex flow, modulated wavy vortex flow, and turbulent Taylor vortex flow [25, 26, 27, 28].

Although a large literature concerning single phase Taylor vortex flow has been built over many years, far less is understood concerning multiphase Taylor vortex flow, particularly with respect to mass transport in such systems. The addition of a second fluid phase leads to instabilities and flow patterns not observed in single-phase flow (e.g. phase inversions and nonhomogeneous distribution of fluid phases) [29, 30, 31, 32, 33]. The available information for interphase mass transport in two-phase Taylor-Couette flow is even more limited, and until now, no computational or theoretical models for interphase mass transfer have been developed for this system. Most of what has previously been reported concerning interphase mass transfer in Taylor-Couette flow is attributable to work performed by Wroński et al. [14] and Dluska et al. [15, 16], who carried out ex- 


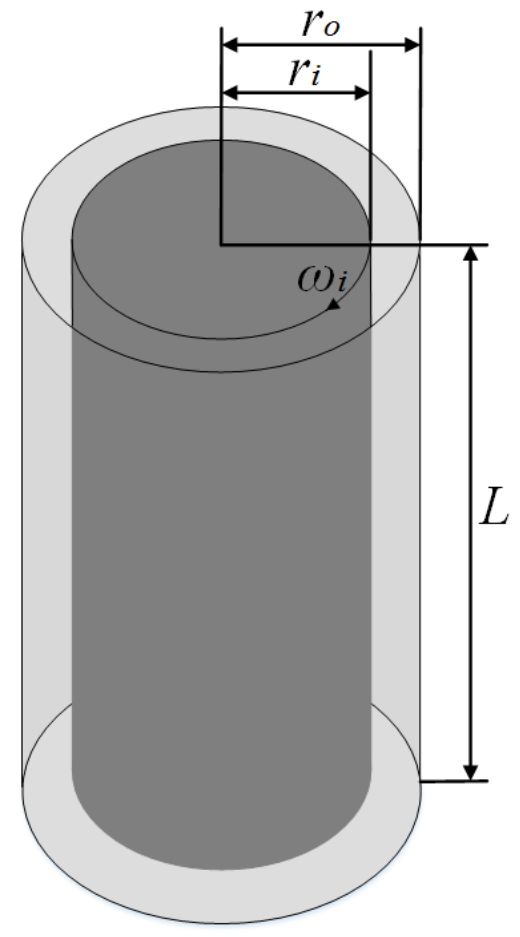

Figure 2: Configuration of a Taylor vortex reactor.

periments in a continuously-fed horizontally-oriented gas-liquid Taylor-Couette reactor and observed volumetric mass transfer coefficients with values on the order of $0.1 \mathrm{~s}^{-1}$. However, the flow patterns generated in a horizontal gas-liquid reactor are significantly different from those that are produced in a vertically oriented reactor, because axial symmetry is destroyed in a horizontal reactor by the vertical gravity field that gives rise to a nonaxisymmetric bouyant force.

In contrast to horizontally oriented gas-liquid Taylor-Couette reactors, the buoyant force acting on gas bubbles is parallel to the cylinder axis in vertically oriented reactors. As a result, effluent gas can easily be separated from the 
liquid phase by feeding gas through the bottom of the reactor and by providing sufficient head space for bubbles to rupture as they emerge from the liquid free surface at the top of the reactor. Such a configuration is particularly useful for delivery of carbon dioxide and removal of oxygen during the culture of phototrophic microorganisms. Interest in vertically oriented gas-liquid Taylor vortex reactors has also been driven by the discovery of the existence of nontrivial bubble distributions and dramatic drag reduction on the rotating inner cylinder $[34,35,36,37]$.

Recently the authors carried out oxygen transport experiments in a vertical gas-liquid Taylor-Couette reactor [38]. They found that gas-liquid mass transfer coefficients in the vertical reactor were significantly smaller than those reported for horizontal reactors. In addition, the authors developed empirical correlations for the mass transfer coefficient and the mean bubble diameter as functions of the liquid azimuthal Reynolds number and the gas axial Reynolds number. While these correlations are useful for understanding the relative contributions of the azimuthal and axial flows in determining the magnitude of mass transfer coefficients, they cannot easily be generalized because (a) Taylor vortex flow patterns cannot be predicted based solely upon axial and azimuthal Reynolds numbers (they also depend upon reactor geometry) and (b) Taylor vortex flow is known to exhibit flow pattern multiplicity, depending upon flow history [25].

In view of the above discussion, it is evident that the prediction of interphase mass transport coefficients for arbitrary Taylor-Couette reactor geometries and operating conditions requires an approach that incorporates details of the fluid flow. To that end, and by making use of our recently-developed computational fluid dynamics simulations for two-phase Taylor vortex flow [39, 40], in this work we compute interphase mass transfer coefficients by integrating local fluid velocity and phase distribution information into well-known theoretical models for interfacial mass transport. This method for computing mass transfer coefficients is then validated by comparing model predictions against our existing experimental data for interphase mass transport in a vertical Taylor-Couette gas-liquid reactor [38]. Indeed, by properly selecting the mass transport model 
appropriate to the fluid flow regime, we demonstrate that this computational approach is capable of reproducing experimental data with high accuracy. Consequently, by making use of information obtained from the computational fluid dynamics (CFD) simulations that is difficult to obtain experimentally, we are able to identify the mechanisms that most strongly impact interphase mass transfer in vertical gas-liquid Taylor-Couette devices.

The remainder of the paper is organized as follows. In Sections 2 and 3 we present the model equations and computational details for both the CFD simulations and the mass transfer coefficient calculations. In Section 4 we validate the computational approach for the prediction of interphase mass transport coefficients by comparing simulation results with experimental data. The computational results are also discussed in greater detail, including consideration of factors such as gas spatial distribution and fluid flow in regions with high bubble concentrations. General conclusions are presented in Section 5 .

\section{Model equations}

\subsection{CFD model for bubbly Taylor-Couette flow}

In this work, the two-phase fluid flow was modeled using an Eulerian-Eulerian approach that we recently employed and validated for bubbly Taylor-Couette flow [39]. The flow was assumed to be axisymmetric [39, 40, 41] so that the quasi-two-dimensional mass, momentum, and species transport equations include an axisymmetric azimuthal velocity component:

$$
\begin{gathered}
\frac{\partial}{\partial t}\left(\alpha_{k} \rho_{k}\right)+\nabla \cdot\left(\alpha_{k} \rho_{k} \vec{u}_{k}\right)=S_{p k} \\
\frac{\partial}{\partial t}\left(\alpha_{k} \rho_{k} \vec{u}_{k}\right)+\nabla \cdot\left(\alpha_{k} \rho_{k} \vec{u}_{k} \vec{u}_{k}\right)=-\alpha_{k} \nabla p+\nabla \cdot\left(\overline{\bar{\tau}}_{k}+\overline{\bar{\tau}}_{k}^{\mathrm{Re}}\right) \\
+S_{p k} \vec{u}_{p k}+\alpha_{k} \rho_{k} \vec{g}+\vec{F}_{I k} \\
\frac{\partial}{\partial t}\left(\alpha_{k} \rho_{k} Y_{k}^{i}\right)+\nabla \cdot\left(\alpha_{k} \rho_{k} \vec{u}_{k} Y_{k}^{i}\right)=-\nabla \cdot\left(\alpha_{k} \vec{J}_{k}^{i}\right)+S_{p k}^{i}
\end{gathered}
$$


Here, $\alpha_{k}$ and $\vec{u}_{k}$ are the phase volume fraction and the phase velocity, respectively. $Y_{k}^{i}$ is the mass fraction of species $i$ in phase $k$. The term $S_{p k}$ is the rate of mass transfer from phase $p$ to phase $k$. The phase stress and Reynolds stress tensors are represented by $\overline{\bar{\tau}}_{k}$ and $\overline{\bar{\tau}}_{k}^{\mathrm{Re}}$, respectively. $J_{k}^{i}$ and $S_{p k}^{i}$ represent the diffusive flux and the net rate of appearance of species $i$ due to interphase mass transfer. The term $\vec{F}_{k}$ is the interphase momentum exchange term, which can be decomposed into at least five independent interphase forces [39, 40, 42]:

$$
\vec{F}_{I k}=\vec{F}_{D}+\vec{F}_{L}+\vec{F}_{V M}+\vec{F}_{W}+\vec{F}_{T}
$$

These interphase forces represent drag, lift, virtual mass, wall lubrication, and turbulent dispersion, respectively. The constitutive relations and model constants are summarized in Tables 1 and 2, respectively. The rationale for the selection of these five interphase forces is as follows. In a recent report on simulation of turbulent gas-liquid Taylor-Couette flow, it was demonstrated that the radial bubble distribution depends primarily upon the competition between the radial pressure gradient, the turbulent dispersion force, and to a lesser extent wall lubrication forces [39]. However, because the axial motion of bubbles is driven by buoyancy, it can be expected that the same interphase forces important for accurately simulating bubble columns, namely drag, virtual mass, and lift forces, must also be included in the analysis [43]. A fuller discussion of various interphase force terms can be found in the recent review article by Buffo and Marchisio [44].

The source terms attributable to interphase mass transport, $S_{p k}$, depend upon the specific physical system under consideration. In this work we seek to validate our computational approach by comparing simulation predictions with our experiments previously reported [38]. In the experiments, the feed liquid and gas phases consisted of water and a binary mixture of oxygen and nitrogen in proportions that approximate the composition of dry air, and the rate of transport of oxygen from the gas bubbles to the water phase was determined from temporal measurements of dissolved oxygen concentration in the liquid phase. Similarly, in this simulation work bubbles are assumed to be dry and the 
Table 1: Constitutive relations for bubbly Taylor-Couette two-fluid model [39, 40, 42].

\begin{tabular}{|l|c|}
\hline drag force [45] & $\begin{array}{c}\vec{F}_{D g}=-\vec{F}_{D l}=\frac{3}{4} \alpha_{g} \alpha_{l} \frac{\rho_{l}}{d_{b}} C_{D}\left|\vec{u}_{g}-\vec{u}_{l}\right|\left(\vec{u}_{l}-\vec{u}_{g}\right) \\
C_{D}=\max \left(\min \left(\frac{24}{\operatorname{Re}_{b}}\left(1+\operatorname{Re}_{b}^{0.687}\right), \frac{72}{\operatorname{Re}}\right), \frac{8 E o}{3(E o+4)}\right)\end{array}$ \\
\hline lift force [46] & $\vec{F}_{L g}=-\vec{F}_{L l}=-C_{L} \alpha_{g} \rho_{l}\left(\vec{u}_{l}-\vec{u}_{g}\right) \times\left(\nabla \times \vec{u}_{l}\right)$ \\
\hline virtual mass force [47] & $\vec{F}_{V M g}=-\vec{F}_{V M l}=-C_{V M} \alpha_{g} \rho_{l}\left[\frac{d}{d t}\left(\vec{u}_{l}-\vec{u}_{g}\right)+\left(\vec{u}_{l} \cdot \nabla \vec{u}_{l}-\vec{u}_{g} \cdot \nabla \vec{u}_{g}\right)\right]$ \\
\hline wall lubrication force [47] & $\vec{F}_{W g}=-\vec{F}_{W l}=-\frac{\alpha_{g} \rho_{l}\left|\vec{u}_{g}-\vec{u}_{l}\right|^{2}}{d_{b}} \max \left[0,\left(C_{1}+C_{2} \frac{d_{b}}{y}\right)\right] \vec{n}_{r}$ \\
\hline turbulent dispersion force $[7,48,49]$ & $\vec{F}_{T g}=-\vec{F}_{T l}=-\rho_{l} \bar{u}_{g}^{\prime} u_{l}^{\prime} \nabla \alpha_{g}=-C_{T} \rho_{l} k_{l} \nabla \alpha_{g}$ \\
\hline
\end{tabular}

Table 2: Constants in bubbly Taylor-Couette two-fluid model [39, 40].

\begin{tabular}{ccccc}
\hline$C_{L}$ & $C_{V M}$ & $C_{1}$ & $C_{2}$ & $C_{T}$ \\
0.02 & 0.5 & -0.01 & 0.05 & 2 \\
\hline
\end{tabular}


effect of water vapor on bubble physical properties and interfacial mass transfer is neglected because the saturation pressure of water vapor in the bubbles at room temperature $(2310 \mathrm{~Pa})$ is much smaller than the partial pressures of oxygen and nitrogen. Hence only the interphase transport of oxygen and nitrogen was considered at the bubble-liquid interface, governed by the following equation:

$$
R_{g l}^{i}=k_{L} a\left(c_{l, *}^{i}-c_{l}^{i}\right), \quad i=\mathrm{O}_{2}, \mathrm{~N}_{2}
$$

where $R_{g l}^{i}$ is the interphase mass transfer rate of species $i, c_{l, *}^{i}=p_{i} / H_{i}, H_{i}$ is the Henry's Law constant for species $i, k_{L} a$ is the volumetric mass transfer coefficient, and $a$ is the specific interfacial area,

$$
a=\frac{6 \alpha_{g}}{d_{b}\left(1-\alpha_{g}\right)} .
$$

Thus, the total volumetric mass transfer rate can be expressed as

$$
S_{g l}=\sum_{i=1}^{I} S_{g l}^{i}=-\sum_{i=1}^{I} \alpha_{g} R_{g l}^{i} M_{w}^{i},
$$

where $M_{w}^{i}$ is the molecular weight of species $i$. Although tracking of interphase nitrogen transport was included for compehensiveness, simulations performed with and without nitrogen transport demonstrated that no difference was found in the predictions, due to the low solubility of nitrogen in water. Consequently it is not necessary to include interphase nitrogen transport in the model equations. Lastly, we note that care must be taken in the use of Eq. (6) for bubble populations with non-monodisperse distributions. In such cases, it is necessary to use the Sauter mean bubble diameter for $d_{b}$.

\subsection{Turbulence modeling}

In this work, the standard $k-\omega$ model was employed to simulate turbulence, as this method has been shown to accurately predict velocity fields, global gas holdup, and the spatial bubble distribution in turbulent gas-liquid TaylorCouette flow [39]. Only liquid phase turbulence is considered, since the bubble phase volume fraction is very low $(<2 \%)$. Indeed, we performed simulations that include bubble induced turbulence (by embedding the Sato bubble induced 
turbulence model into the standard turbulence model - see Sato and Sekoguchi [50] for details) and found no significant difference in the predictions compared to cases simulated without bubble induced turbulence. For the same reason, bubble-bubble interactions were also neglected. The turbulent kinetic energy $\left(k_{l}\right)$ and the specific dissipation rate $\left(\omega_{l}\right)$ are governed by the following transport equations [51]:

$$
\begin{gathered}
\frac{\partial}{\partial t}\left(\alpha_{l} \rho_{l} k_{l}\right)+\nabla \cdot\left(\alpha_{l} \rho_{l} k_{l} \vec{u}_{l}\right)=\nabla \cdot\left(\alpha_{l}\left(\mu_{l}+\frac{\mu_{l, t}}{\sigma_{k}}\right) \nabla k_{l}\right)+\alpha_{l}\left(G_{k}-Y_{k}\right) \\
\frac{\partial}{\partial t}\left(\alpha_{l} \rho_{l} \omega_{l}\right)+\nabla \cdot\left(\alpha_{l} \rho_{l} \omega_{l} \vec{u}_{l}\right)=\nabla \cdot\left(\alpha_{l}\left(\mu_{l}+\frac{\mu_{l, t}}{\sigma_{\omega}}\right) \nabla \omega_{l}\right)+\alpha_{l}\left(G_{\omega}-Y_{\omega}\right)
\end{gathered}
$$

where $G_{k}$ and $Y_{k}$ represent the generation and dissipation of turbulent kinetic energy, and $G_{\omega}$ and $Y_{\omega}$ represent the generation and dissipation of $\omega$, respectively. The terms $\sigma_{k}$ and $\sigma_{\omega}$ represent the turbulent Prandtl numbers for $k$ and $\omega$, respectively, and are both taken to be equal to 2.0. The turbulent viscosity $\mu_{l, t}$ depends upon $k_{l}$ and $\omega_{l}$ as follows:

$$
\mu_{l, t}=\frac{\rho_{l} k_{l}}{\omega_{l}} .
$$

\subsection{Mass transfer models}

Commonly employed models for the prediction of the liquid-side mass transport coefficient for gas bubbles in a continuous liquid phase include the penetration [52], eddy cell [53] and laminar boundary layer models [54]. Thorough overviews of these and other models can be found in Huang et al. [7] and GarciaOchoa and Gomez [? ]. The laminar boundary layer model is written as

$$
k_{L}=C \sqrt{\frac{u_{\text {slip }}}{d_{b}}} D_{L}^{2 / 3} \nu_{l}^{-1 / 6},
$$

where the slip velocity is defined as $u_{\text {slip }}=\left|\vec{u}_{g}-\vec{u}_{l}\right|, d_{b}$ is the bubble diameter, $D_{L}$ is the diffusivity of the solute in the liquid phase, $\nu_{l}$ is the kinematic viscosity of the liquid, and $C$ is a constant. Different values of $C$ have been reported including $0.6[7,55]$ and $0.42[54,56]$. The laminar boundary layer model can 
be applied only for rigid bubbles with small diameter $\left(d_{b}<1 \mathrm{~mm}\right)$ or bubbles with completely immobile surface due to contamination [57]. Some authors have found that the laminar boundary layer model underpredicts the mass transfer coefficient when it is applied to bubble columns or stirred tanks [7].

The penetration theory proposed by Higbie [52] assumes that bubble surfaces are immobile, but it accounts for unsteady diffusion of gas into the liquid at the gas-liquid interface by introducing a characteristic gas-liquid exposure time, leading to the following expression:

$$
k_{L}=\sqrt{\frac{4 D_{L}}{\pi t_{e}}} .
$$

An estimate is needed for the exposure time, given by $t_{e}=d_{b} / F^{2} u_{\text {slip }}$, where $u_{\text {slip }}$ is the slip velocity, $d_{b}$ is the bubble diameter, and where $F$ is a constant. Substitution of the exposure time into Eq. 12 leads to

$$
k_{L}=F \sqrt{\frac{4 D_{L} u_{\text {slip }}}{\pi d_{b}}} .
$$

In the Higbie penetration theory for a single bubble, the constant $F$ is taken as unity, whereas smaller values have been used by investigators when considering bubble swarms and high gas volume fractions. For instance, Wang et al. [58] found that the volumetric mass transfer rate in a bubble column can be well predicted using $F=0.8$.

The penetration theory model is known to underpredict mass transfer for highly turbulent flows, and Eq. 12 implies that this is caused by an overestimation of the exposure time. Indeed, several experimental studies of gas-liquid transport in turbulent flow have revealed that the liquid-side mass transfer is controlled by interfacial surface renewal by small scale eddies rather than by any measure of the mean flow of the liquid relative to the bubbles, such as the slip velocity [53]. As a result, for highly turbulent flow it is more appropriate to estimate the characteristic gas-liquid exposure time using the Kolmogorov theory of isotropic turbulence, which leads to the following "eddy cell model":

$$
k_{L}=K \sqrt{\frac{4 D_{L}}{\pi}\left(\frac{\varepsilon_{l}}{\nu_{l}}\right)^{1 / 2}}=K^{\prime} \sqrt{D_{L}\left(\frac{\varepsilon_{l}}{\nu_{l}}\right)^{1 / 2}} .
$$


In the above equation, $\varepsilon_{l}$ represents the liquid turbulence dissipation rate. Various values of the model constant $K^{\prime}=(4 / \pi)^{1 / 2} K$ have been suggested in the literature, e.g. $(4 / \pi)^{1 / 2}$ [5], 0.592 [57], 0.4 [53], 0.301 [6], 0.523 [56], 0.27 [58]. Wang [58] has hypothesized that the inconsistency of the various estimates for $K^{\prime}$ stem from two causes including (1) the difficulty in accurately calculating turbulent dissipation rates and (2) lack of consideration of the bubble swarm effect on mass transfer.

Although the various mass transfer models discussed above find wide usage, none are appropriate for simulating all of the local conditions and flow patterns that arise in Taylor-Couette devices. Specifically, the assumptions associated with the classical penetration theory model are inconsistent with turbulent flow and lead to an overestimation of the exposure time at high cylinder rotation speeds, whereas the eddy cell model overpredicts exposure time for low dissipation rates at low rotation speeds. For this reason, we introduce an adaptive mass transfer model that combines the strengths of both models and that can be used over a wide range of Reynolds numbers and at all locations in the reactor. Specifically, by computing the local exposure time for both models, the most appropriate model for given flow conditions can be selected by choosing the one that predicts the smallest exposure time. i.e.,

$$
t_{e}=\min \left(\frac{1}{F^{2}} \frac{d_{b}}{u_{\text {slip }}}, \frac{1}{K^{2}} \sqrt{\frac{\nu_{l}}{\varepsilon_{l}}}\right) .
$$

Here, $F$ and $K$ are adjustable constants that are needed to account for the fact that the slip velocity is sensitive to the drag model used and the turbulence dissipation rate is sensitive to the turbulence model employed. Consequently, the adaptive mass transfer model used in this work can be expressed as follows:

$$
k_{L}=\max \left(F \sqrt{\frac{4 D_{L}}{\pi} \frac{u_{\text {slip }}}{d_{b}}}, K \sqrt{\frac{4 D_{L}}{\pi}\left(\frac{\varepsilon}{\nu_{l}}\right)^{1 / 2}}\right) .
$$

In order to make use of the penetration model, the eddy cell model, or the adaptive model described above, it is necessary to choose values for the model constants $F$ and $K$. Although the range of previously reported values for $F$ 
and $K$ is not large, we have chosen to fit these parameters by making use of measurements reported in our experimental investigation of oxygen mass transport in gas-liquid Taylor-Couette [38], which is described in more detail in Section 4.1. After testing several values for both $F$ and $K$, these constants were taken to be $F=1.2$ and $K=0.35$. These values are consistent with those used by other investigators, as was discussed previously.

\section{Simulation approach and computational details}

\subsection{Overview of computational approach}

As was previously mentioned, the overall strategy for computing interphase mass transfer coefficients consists of (1) performing two-phase CFD simulations in order to compute instantaneous and time-averaged velocity fields and fluid phase distributions, and (2) computing local interphase mass transfer coefficients $k_{L}$ from Eq. 16 by using flow field predictions to estimate turbulence energy dissipation rates $(\varepsilon)$ and slip velocities $\left(u_{\text {slip }}=\left|\vec{u}_{g}-\vec{u}_{l}\right|\right)$. It should be noted that the Eulerian CFD approach used here requires foreknowledge of the mean gas bubble diameter, as this quantity is needed to compute components of the interfacial momentum exchange rate $\vec{F}_{k}$, such as drag and virtual mass. Furthermore, once the mean bubble diameter is specified and the resulting gas holdup is computed, the specific surface area can also be computed from Eq. 6, and subsequently interphase mass transfer rates can be determined. For this reason, and also to validate the computational approach with experiments, the simulations reported here were performed using the same geometry and fluids corresponding to the experimental conditions used by Ramezani et al. [38], who developed the following correlations for the volumetric mass transfer coefficient and mean bubble size in a vertical gas-liquid Taylor-Couette reactor:

$$
\begin{gathered}
\mathrm{Sh}=\frac{k_{L} a d_{b}^{2}}{D_{L}}=1.45 \times 10^{-7} \operatorname{Re}_{a}^{0.83}\left(\operatorname{Re}_{\theta}+1.47 \times 10^{4}\right)^{1.61} \\
\frac{d_{b}}{r_{o}-r_{i}}=1.0 \times 10^{-5} \operatorname{Re}_{a}^{0.11}\left(\operatorname{Re}_{\theta}+3.3 \times 10^{4}\right)^{0.91}
\end{gathered}
$$


In the above expressions $d_{b}$ is the Sauter mean diameter, $\operatorname{Re}_{a}$ is the axial Reynolds number based upon the gas flow rate, and $\operatorname{Re}_{\theta}$ is the azimuthal Reynolds number based upon the liquid phase fluid properties. Hence, the mean bubble sizes needed to carry out the CFD simulations were obtained from Eq. 18, and the resulting simulation predictions for $k_{L}$ were compared against those given by Eq. 17 .

\subsection{Computational domain and boundary conditions}

A schematic of the Taylor-Couette apparatus used in the experiments of Ramezani et al. [38] and in the simulations presented here is shown in Fig. 3. The rotating inner cylinder has an outer radius of $3.81 \mathrm{~cm}$ and the fixed transparent acrylic outer cylinder has an inner radius of $5.08 \mathrm{~cm}$, resulting in a gap width of $1.27 \mathrm{~cm}$. The length of the reactor is $50.8 \mathrm{~cm}$ and the reactor is filled to a height of $h=48 \mathrm{~cm}$, thus the total liquid working volume within the reactor is $1.70 \mathrm{~L}$. The corresponding radius ratio and aspect ratio of the apparatus are given by $\eta=r_{i} / r_{o}=0.75$ and $\Gamma=h /\left(r_{o}-r_{i}\right)=40$, respectively.

A non-uniform rectangular mesh was employed to simulate the annular flow chamber, and three progressively finer mesh resolutions $(20 \times 240,32 \times 480,50 \times$ 720) radial $\times$ axial nodes were tested until a grid-independent solution was obtained. Because no differences were observed in the solution between the two finest grids tested, all subsequent computations were performed using the $32 \times 480$ mesh. It should be noted that in the near-wall regions a finer grid spacing was used ( 8 grid points within $2.5 \mathrm{~mm}$ of either wall) to capture steep gradients associated with the no-slip boundary conditions. The first layer of grid points was located within the viscous sublayer at wall units $y^{+}=3.3$ and 5.0 for the inner and outer cylinders, respectively, and the mesh is depicted graphically in Figure S1 in the Supplemental Information.

The reactor operated in a semi-batch mode with continuous flow of gas and no feed or removal of liquid. The gas inlet was modeled as a narrow channel with a width of $10 \mathrm{~mm}$ near the outer wall at the bottom of the reactor to mimic the feed conditions in the experiment. The boundary at the top of the annular 


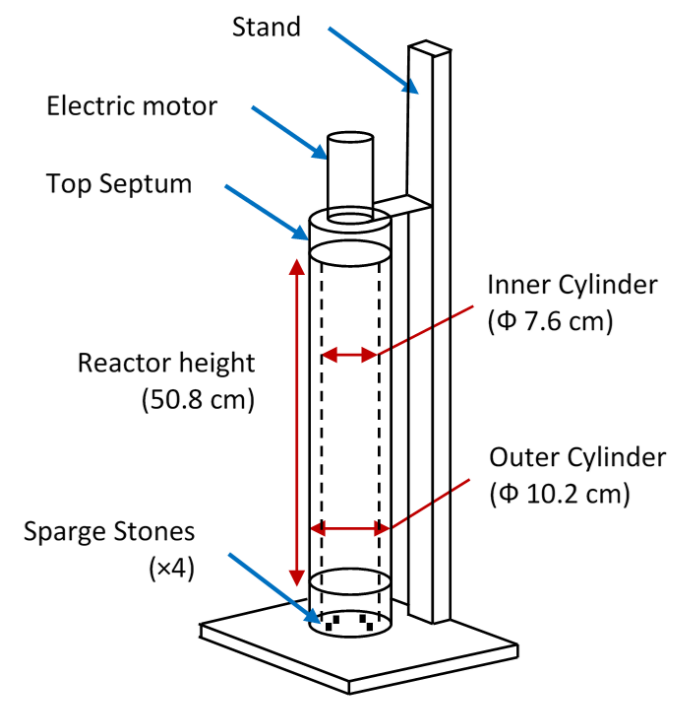

Figure 3: Schematic of Taylor-Couette bioreactor used for mass transfer experiments [38].

fluid mixture was treated as a gas outflow boundary to simulate the liquid free surface in contact with a head space. In summary, the boundary conditions used were as follows. No-slip conditions were used at solid boundaries, and the azimuthal velocity of the inner cylinder was chosen to match experimental conditions. A fixed gas velocity was prescribed at the gas inlet at the bottom of the reactor and by specifying an inlet bubble volume fraction of 0.05 . Pressure outlet boundary conditions were used for both fluid phases.

Simulations were initialized assuming that only the liquid phase occupied the annular flow chamber so that $\alpha_{g}=0$. Subsequently, the momentum, mass, and species conservation equations described above were solved in double precision using the commercial finite volume CFD code, ANSYS FLUENT 14.5 (Ansys Inc., US). The interphase mass transfer model was embedded into the CFD code with the aid of user defined functions (UDFs). The pressure-velocity coupling 
was resolved using the SIMPLE algorithm [59]. Transient CFD simulations were carried out using a time step of $5 \times 10^{-4} \mathrm{~s}$. Generally $30-60 \mathrm{~s}$ of simulated physical time was required to reach a quasi steady state wherein the global gas volume fraction did not change with time.

\section{Results and Discussion}

In our previous work [39], the two-fluid model described in Section 2 was validated by direct comparison of simulated results with velocity and phase distribution data from experiments performed by Murai et. al. [60], who carried out studies of a vertical semibatch gas-liquid Taylor-Couette reactor. Consequently, we focus here on model predictions for mass transfer calculations.

\subsection{Selection of Mass Transfer Model Parameters}

As was mentioned in Section 2.3, the penetration, eddy cell, and adaptive mass transfer models require specification of the model constants $F$ and $K$. The values used here $(F=1.2, K=0.35)$ were obtained by fitting simulation predictions for the penetration and eddy cell models to data from corresponding experiments described in our earlier work [38]. Specifically, in order to select the optimal value of $F$, simulations of gas-liquid oxygen mass transfer using the penetration model (13) were performed for several values of $F$ and for the lowest rotation speed considered, and the volumetric averaged values of $k_{L} a$ were compared with experimentally determined values. A similar procedure was used to select the optimal value of $K$, except that simulations were performed using the eddy cell mass transfer model (14) and compared with data from experiments performed at the highest rotation speeds considered.

\subsection{Validation of Adaptive Mass Transfer Model}

Axially averaged plots of the radial distribution of the simulated volumetric mass transfer coefficient using different theoretical models are shown in Fig. 4 (a). Specifically, calculations were performed using the laminar boundary layer (Eq. 11), penetration (Eq. 13), eddy cell (Eq. 14), and adaptive (Eq. 16) models. 
It can be seen from these plots that interphase mass transfer is virtually nonexistent in the reactor except near the inner cylinder, because that region is where most of the gas is located (Fig. 4 (b)).
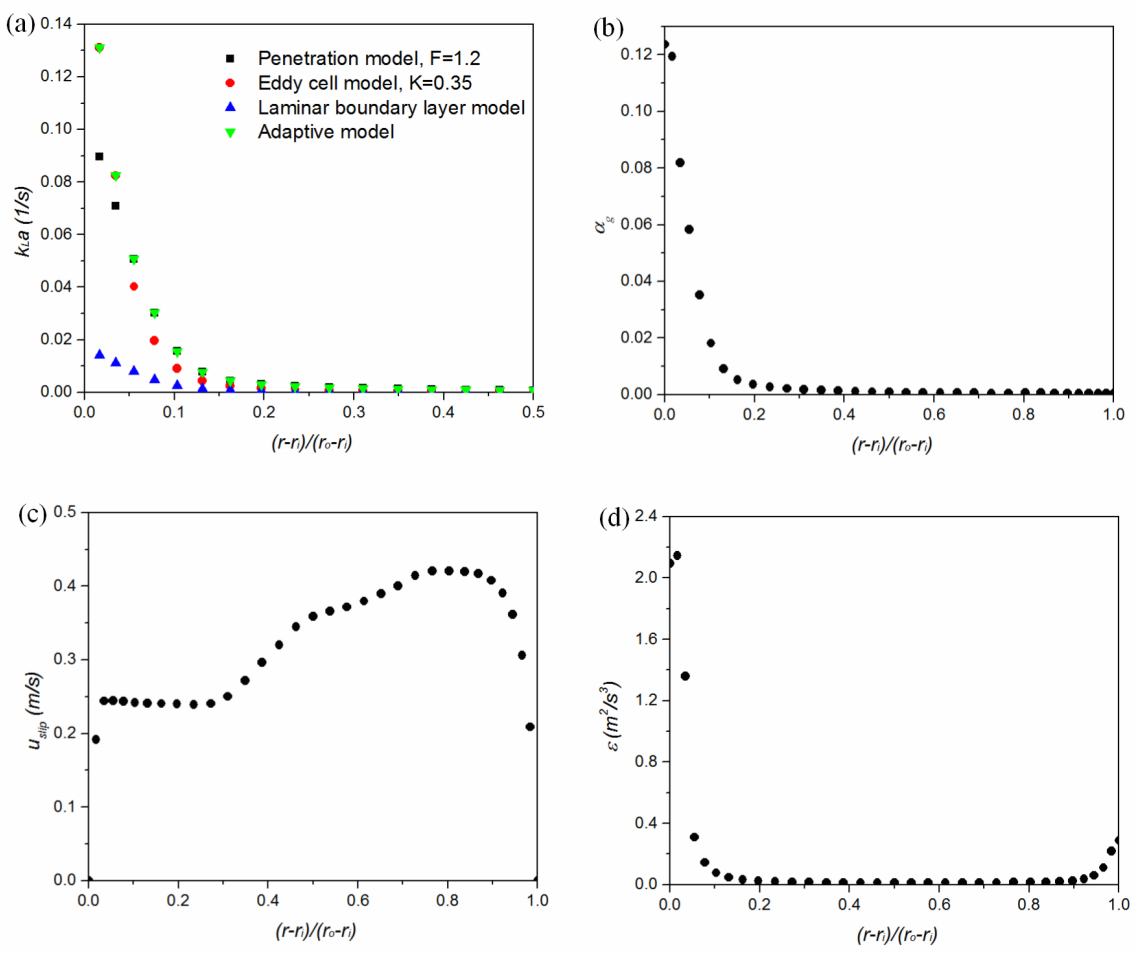

Figure 4: Axially averaged radial distributions of (a) volumetric mass transfer coefficient $(1 / \mathrm{s})$, (b) gas volume fraction, (c) slip velocity $(\mathrm{m} / \mathrm{s})$, and (d) turbulence dissipation rate $\left(\mathrm{m}^{2} / \mathrm{s}^{3}\right)$ for rotation speed and gas flow rate of $300 \mathrm{rpm}$ and $0.235 \mathrm{vvm}$, respectively.

Comparison of the simulated spatially averaged volumetric mass transfer coefficient with experimental data is shown in Figs. 5 and 6 for various rotation speeds and gas flow rates. Examination of the data presented in Fig. 5 suggests that $k_{L} a$ is relatively insensitive to rotation speed for speeds lower than $300 \mathrm{rpm}$, but increases monotonically at higher rotation speeds. In contrast, Fig. 6 demonstrates that the volumetric mass transfer coefficient increases 

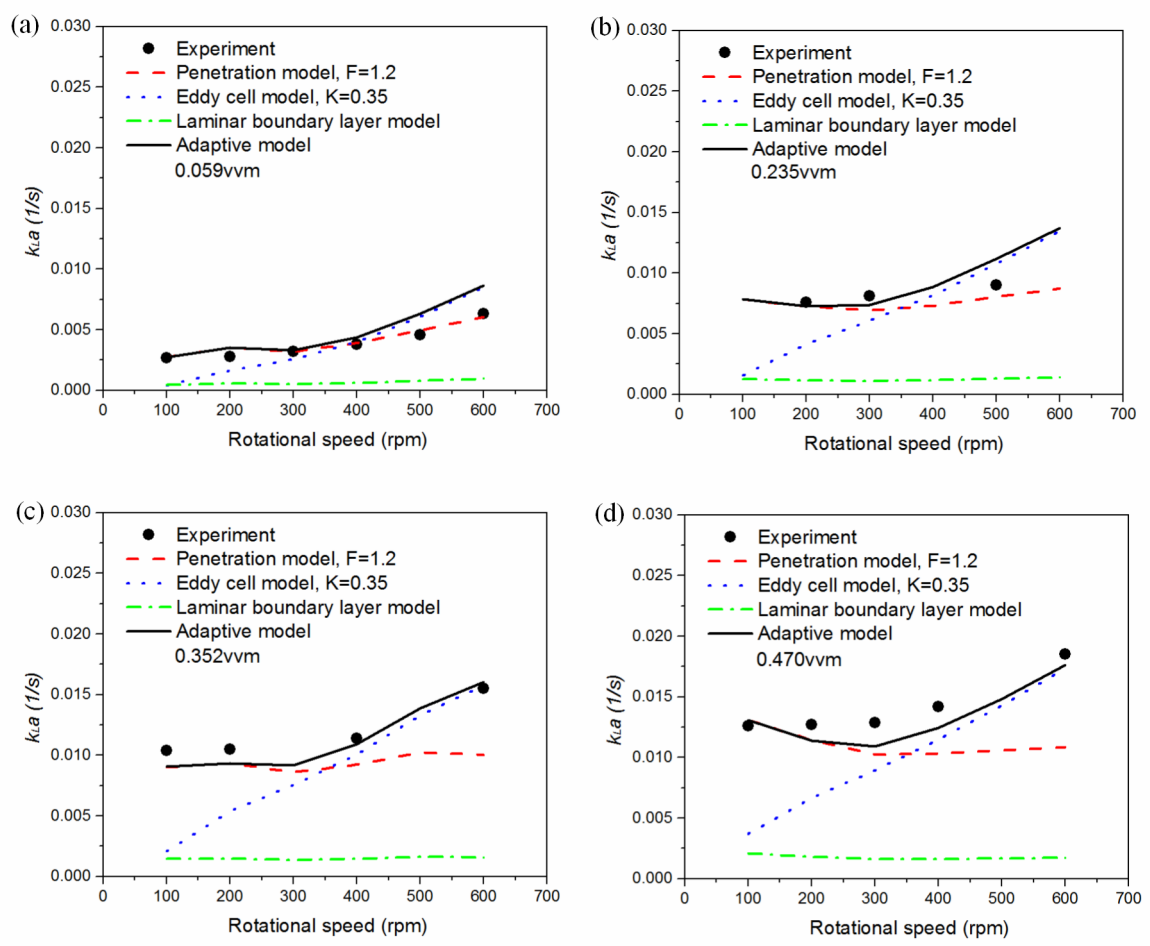

Figure 5: Comparison of predicted average volumetric mass transfer rate by different mass transfer models with experimental data under different rotational speeds.

with increasing gas flow rate over the entire range of cylinder speeds studied. In all cases considered, mass transfer coefficients computed using the laminar boundary layer model are significantly lower than the corresponding experimental values, as is expected since the flow conditions do not satisfy the laminar boundary layer model assumptions. As expected, the penetration model, which relies on slip velocity to estimate characteristic gas-liquid exposure time, produces values of $k_{L} a$ very close to experimental values observed at low rotation speeds, whereas it fails at the higher rotation speeds studied. In contrast, the eddy cell model is more accurate for high cylinder rotation speeds but produces values that are too low at the lower rotation speeds. These results are consistent 

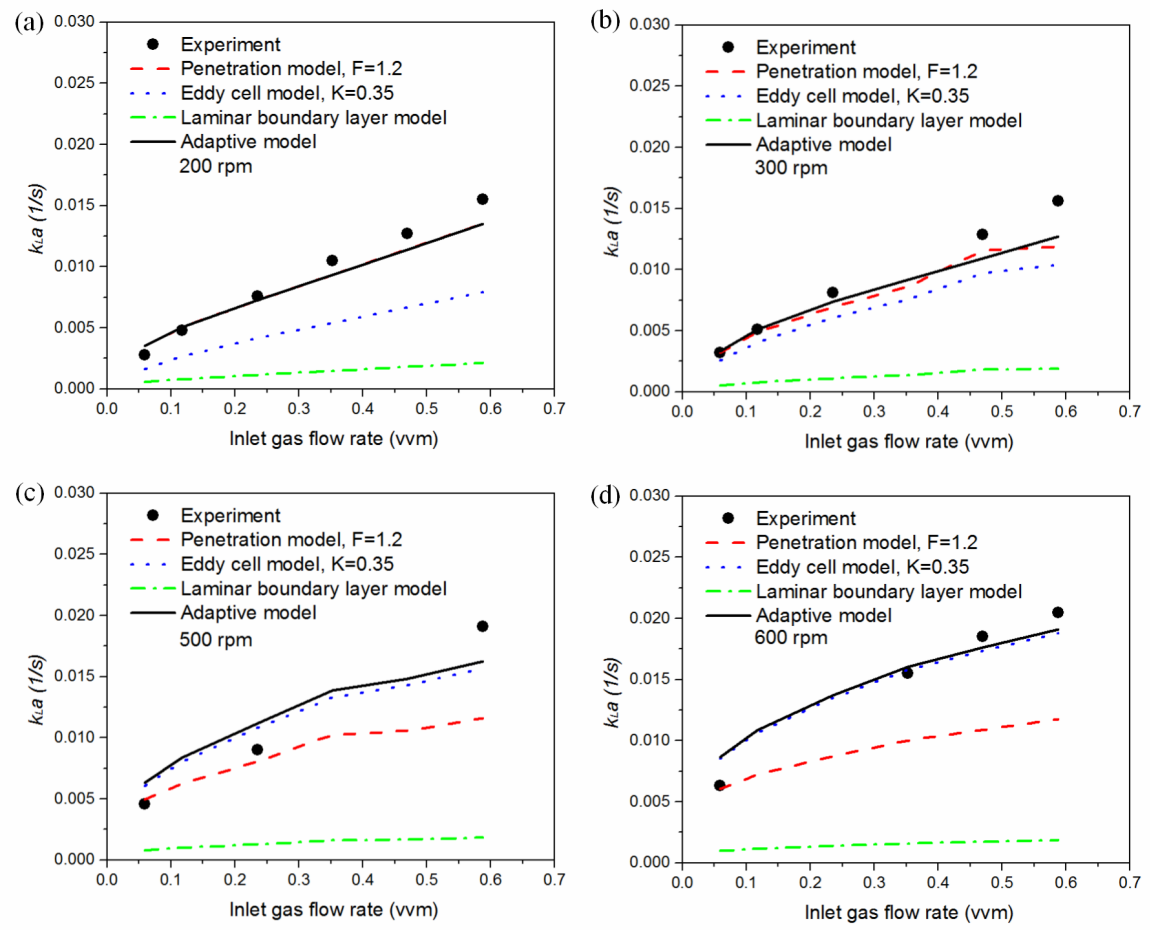

Figure 6: Comparison of predicted average volumetric mass transfer rate by different mass transfer models with experimental data under different inlet gas flow rates.

with findings of Alves et al. [57], who showed that the penetration model can be used to calculate bubble mass transfer coefficients for turbulent dissipation rates up to at least $\varepsilon \approx 0.04 \mathrm{~m}^{2} / \mathrm{s}^{3}$. For the calculations presented in Figs. 5 and 6 , the penetration model becomes inaccurate for spatially averaged turbulent dissipation rates larger than approximately $0.1 \mathrm{~m}^{2} / \mathrm{s}^{3}$. Fortunately, the eddy cell model accurately predicts the volumetric mass transfer coefficient when the turbulent dissipation rate is larger than $0.1 \mathrm{~m}^{2} / \mathrm{s}^{3}$. Consequently, the proposed adaptive mass transfer model (Eq. 16) combines the advantages of both the penetration and eddy cell models in order to produce good global predictions over a wide range of turbulent flow conditions by automatically selecting the most 
appropriate mass transfer model for local conditions. A few exceptions occur at high rotation speeds and low gas flow rates, where the penetration model appears to perform slightly better than the adaptive model. Further experiments under these conditions will be needed in order to determine if the selection criterion of the adaptive model can be improved, for example by accounting for the local gas holdup.

Although the comparison of experimental measurements with predictions based upon the adaptive mass transport model combined with CFD simulations provide evidence for the validity of the modeling approach used here, it is possible to further validate the model by comparing simulation predictions with raw experimental results. For example, in the work of Ramezani et al. [38], values of $k_{L} a$ were determined by measuring dissolved oxygen in the liquid phase in response to a step change in the feed gas composition. Simulation of the evolution of dissolved oxygen concentration for one of these experiments (by including species transport equations in the CFD simulations) is shown in Fig. 7 and compared directly to the experimental measurements. Because of startup transients associated with the experimental protocol (more fully described in [38]), there is a brief induction period before dissolved oxygen is detected by the oxygen sensor. This startup transient is not observed in the simulations because mass transfer was enabled only after the two-phase fluid flow reached a steady state. In spite of these important differences, the predictions are in remarkably good agreement with the experiments.

We also note that Ramezani et al. [38] assumed (and confirmed by experiments) that the bulk liquid phase was sufficiently well mixed so that the dissolved oxygen concentration was essentially spatially uniform and therefore dissolved oxygen measurements at a single point are sufficient for determining the global value of $k_{L} a$. Indeed, the simulation of these experiments predicts spatial homogeneity in the distribution of oxygen in the liquid phase (not shown). 


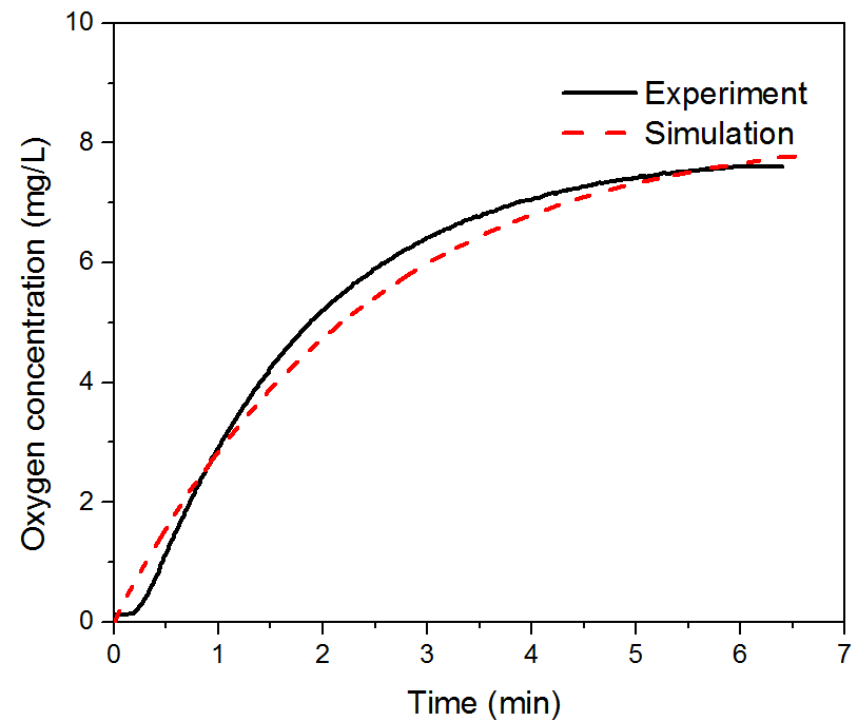

Figure 7: Comparison of simulated instantaneous liquid phase oxygen concentration with experimental data of a specific case (0.235 vvm, $300 \mathrm{rpm})$ at an axial location of $12.7 \mathrm{~cm}$ from the bottom of the reactor and near the outer cylinder wall.

\subsection{Factors influencing mass transfer rate}

For the mass transfer models considered here, $k_{L}$ and $a$ are most directly impacted by slip velocity, dissipation rate and bubble diameter. These quantities in turn are determined by system operating conditions, geometrical parameters, and physical properties, as shown in Fig. 1. Here, we discuss the impact of several factors, including quantities that are difficult to obtain from experiments but that are accessible from CFD calculations.

As was discussed previously, a priori knowledge concerning bubble diameter is required to carry out the computations. Specifically, the Sauter mean bubble diameter was estimated using the empirical correlation given by Eq. 18 [38], 
which depends upon both the azimuthal and axial Reynolds numbers. From this equation, it is evident that the azimuthal Reynolds number (cylinder rotation speed) has a stronger effect on bubble diameter than does the axial Reynolds number (gas flow rate). For example, a five-fold increase in rotation speed produces an increase in bubble diameter of $\approx 50 \%$, whereas a five-fold increase in gas feed rate only results in a bubble diameter increase of $\approx 20 \%$. These dependencies in turn have a direct effect on the specific interfacial area $a$, as well as indirect effects on $k_{L}$ (for example by impacting the bubble drag force and slip velocity).

Figure 4 (b)-(d) shows axially averaged plots for the radial distribution of gas volume fraction, slip velocity, and turbulence dissipation rate for a specific gas flow rate and rotation speed $(0.235 \mathrm{vvm}, 300 \mathrm{rpm})$, but these results are representative of all the conditions studied here. As can be seen in Fig. 4 (c), the largest slip velocities occur near the outer cylinder wall. This phenomenon can be explained by the fact that most of the gas is concentrated near the inner cylinder wall (see Fig. 4 (b)). The rising gas near the inner cylinder entrains liquid, which recirculates toward the bottom of the reactor near the gas-depleted outer cylinder. As a result, the slip velocity near the outer cylinder is quite large, but there are very few bubbles in this region.

The dependence of the volume averaged (global) slip velocity on gas flow rate and cylinder rotation speed is depicted in Fig. 8. Surprisingly, this quantity decreases with increasing gas flow rate. While this result may be counter-intuitive, several factors should be considered when interpreting this result. For example, the slip velocity depends upon the bubble drag. The Tomiyama drag model [45] used here in turn has a very complicated dependence on gas flow rate, particularly as it impacts bubble diameter [38]. In addition, gas flow rate can lead to significant changes in the flow patterns, including stretching the vortex axial wavelength [39].

The global volume averaged turbulent kinetic energy dissipation rate is plotted in Fig. 9 for various rotation speeds and gas flow rates, and it is apparent that this quantity is insensitive to the gas flow rate. While such a result may 

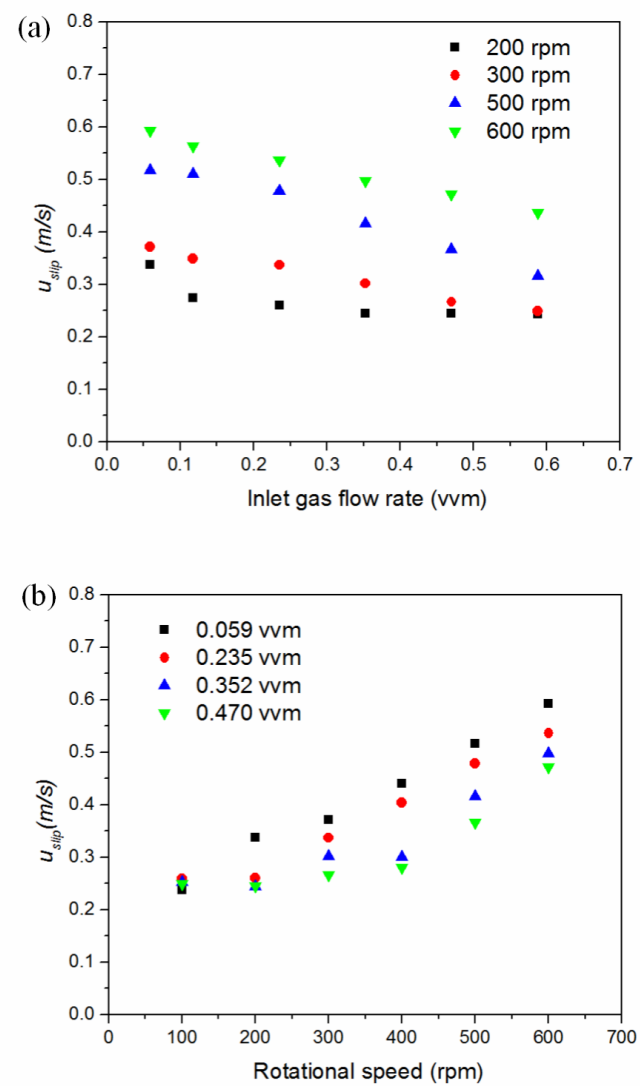

Figure 8: Global slip velocity as a function of inlet gas flow rate and cylinder rotation speed.

not be surprising since the gas phase is neglected in the turbulence model and bubble induced turbulence is not considered (due to the low gas holdup), the presence of gas could be expected to indirectly impact the dissipation rate since the liquid velocity field is influenced by the presence of gas bubbles. In contrast, $\varepsilon$ increases rapidly with cylinder rotation speeds above $300 \mathrm{rpm}$. Furthermore, the turbulence dissipation rate is much larger near the cylinder walls than in the middle of the annulus, as is evident from Fig. 4 (d).

The simulated global gas holdup, which was obtained by spatial averaging, 

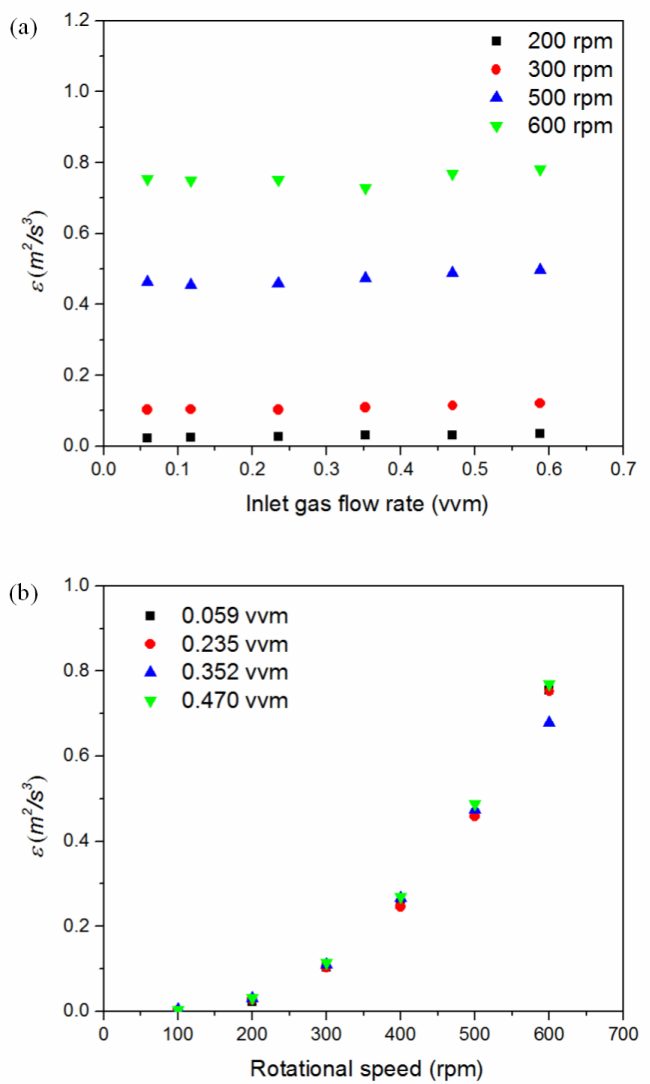

Figure 9: Global turbulent dissipation rate as a function of inlet gas flow rate and cylinder rotation speed.

is shown in Fig. 10 for the case of no cylinder rotation, and these results are compared with experimental data from our previous work [38]. Although the predicted gas holdup agrees well with the experimental data for a wide range of inlet gas flow rates tested, the simulated gas holdup is slightly but consistently lower than the experimental data, particularly at the higher gas flow rates considered. This discrepancy can probably be attributed to one or both of two causes, including (1) errors in estimates of the bubble diameter in Eq. 18 at high gas flow rates, due to the fact that in such cases individual bubbles 
are difficult to distinguish because of the presence of bubble swarms [38] and (2) errors associated with the drag model when bubble swarms are present. Indeed, the Tomiyama [45] drag model used in this study was developed for single bubbles rather than for bubble swarms, and subsequent studies of drag on bubble swarms in bubble columns $[61,62]$ have demonstrated that corrections are required, although no such correction currently exists for gas-liquid Taylor-Couette flow.

For cases with rotation of the inner cylinder (turbulent Taylor-Couette flow), the predicted global gas holdup is shown in Fig. 11 for various gas flow rates and rotation speeds. No experimental data are available for comparison, because the liquid free surface at the top of the reactor has a complex topography when the inner cylinder rotates, and therefore it is difficult to accurately measure global gas holdup. It can be seen from Fig. 11 (a) that for constant cylinder rotation speed, gas holdup increases approximately linearly with the inlet gas flow rate. In contrast, for fixed inlet gas flow rate, the gas holdup is relatively insensitive to rotation speeds below $300 \mathrm{rpm}\left(\operatorname{Re}_{\theta}=15200\right)$, whereas it increases approximately linearly for rotation speeds above this value.

In view of the non-uniform spatial distribution of slip velocity and turbulent kinetic energy dissipation, it is important to understand not only how the global gas holdup depends upon operating parameters, but also how the spatial distribution of gas is affected. Returning to Fig. 4 (a), this plot demonstrates that bubbles concentrate near the inner cylinder wall, driven by the strong centrifugal force [39], as was discussed previously. Note that Fig. 4 (d) shows that this region is also characterized by high dissipation rates. The same results occur for the other flow conditions studied with cylinder rotation, and together these findings demonstrate that interphase mass transport is mainly confined to the region near the inner cylinder, which is discussed further in the next section.

\subsection{Mass transfer coefficients and interfacial area}

Although globally averaged values of $k_{L} a$ were computed in order to compare model predictions to experimental findings, a deeper understanding of the 


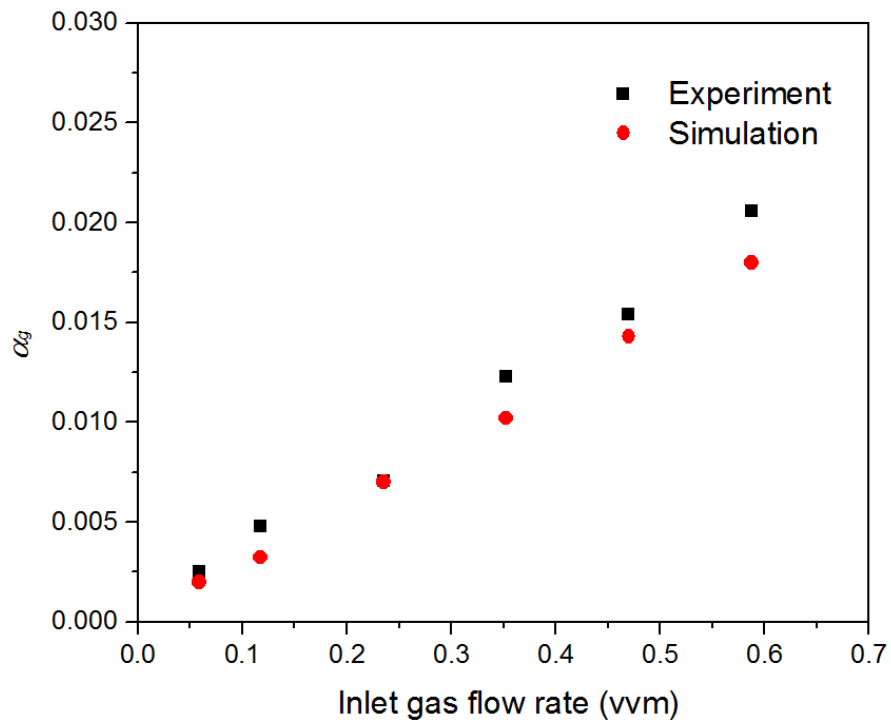

Figure 10: Comparison of simulated volume averaged gas holdup with experimental data for the case of no cylinder rotation.

relationship between this quantity and reactor operating conditions can be obtained by examining the local and global dependencies of the specific interfacial area $a$ and the mass transfer coefficient, $k_{L}$, individually. For example, typical local values of the mass transfer coefficient $k_{L}$ computed using the adaptive model are shown in the spatial contour plot in Fig. 12 (a), whereas the local specific interfacial area $a$, which was computed based upon knowledge of the gas spatial distribution and the mean bubble diameter, is presented in Fig. 12 (b). Figures 12 (a) and (b) demonstrate that the mass transfer coefficient is much more uniformly distributed (although there are enhancements near both cylinders) than is the specific interfacial area, because the latter quantity depends upon the local gas volume fraction. The enhanced values of the mass transfer coefficient near the inner and outer cylinders reflect the corresponding distribu- 

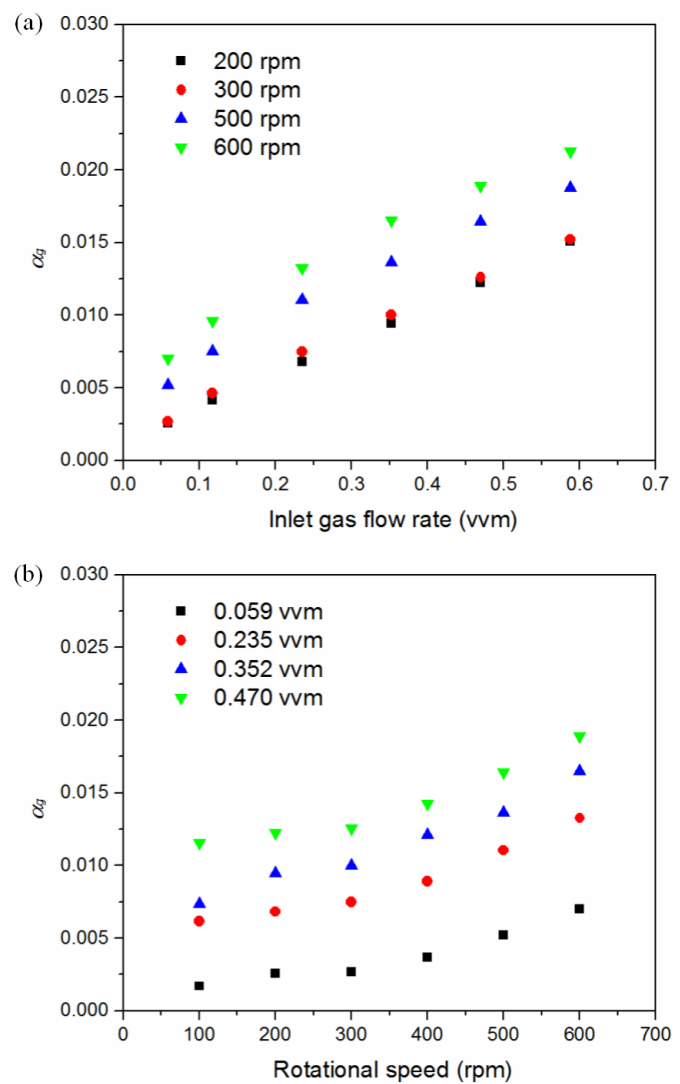

Figure 11: Predicted global volume averaged gas fraction as a function of inlet gas flow rate and rotational speed.

tions of turbulent energy dissipation rate and slip velocity discussed previously and depicted in Fig. 4.

The effect of inlet gas flow rates and rotation speeds on the global spatially averaged values of the mass transfer coefficient and the interfacial area are shown in Figs. 13 and 14. Interestingly, the global mass transfer coefficient decreases with increasing gas flow rate at constant cylinder rotation speed. This result can be understood by considering how increased gas flow rate impacts local mass transfer coefficients, which are computed using Eq. 16. In particular, we note 


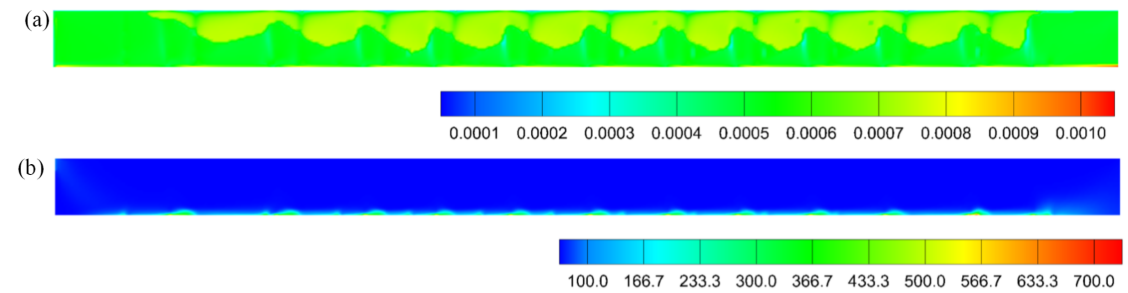

Figure 12: Contour plots of (a) mass transfer coefficient $k_{L}(\mathrm{~m} / \mathrm{s})$ predicted by the adaptive mode and (b) specific interfacial area $a\left(\mathrm{~m}^{2} / \mathrm{m}^{3}\right)$ for a gas flow rate of $0.235 \mathrm{vvm}$ and cylinder rotation speed of $300 \mathrm{rpm}$. In each plot, the inner cylinder wall is represented by the bottom boundary and the outer cylinder wall is represented by the top boundary.

that for regions with high turbulence dissipation rates, $k_{L}$ is governed by the eddy cell model, which is essentially independent of the gas flow rate because the turbulence dissipation rate is insensitive to gas flow rate (Fig. 9 (a)) for the conditions studied. In regions with low dissipation rates, $k_{L}$ is governed by the penetration theory model, which has a more complicated dependence on gas flow rate. Specifically, as was discussed previously the slip velocity decreases while bubble diameter increases with increasing gas flow rate, thereby causing the local value of $k_{L}$ to decrease. Hence, increasing the feed gas flow rate leads to reduced local values of the mass transfer coefficient in regions governed by the penetration theory model and unchanged values in high dissipation rate regions governed by the eddy cell model, thereby leading to an overall decrease in the global mass transfer coefficient.

Fig. 14 demonstrates that specific interfacial area, which depends upon both the gas holdup and bubble diameter, increases rapidly with gas flow rate. In contrast, for cylinder rotation speeds less than $300 \mathrm{rpm}$, the specific interfacial area is insensitive to rotation speed. Above $300 \mathrm{rpm}$, the specific interfacial area increases weakly with rotation speed. In view of the results shown in Figs. 13 and 14, the monotonic increase of the volumetric mass transfer coefficient $k_{L} a$ 

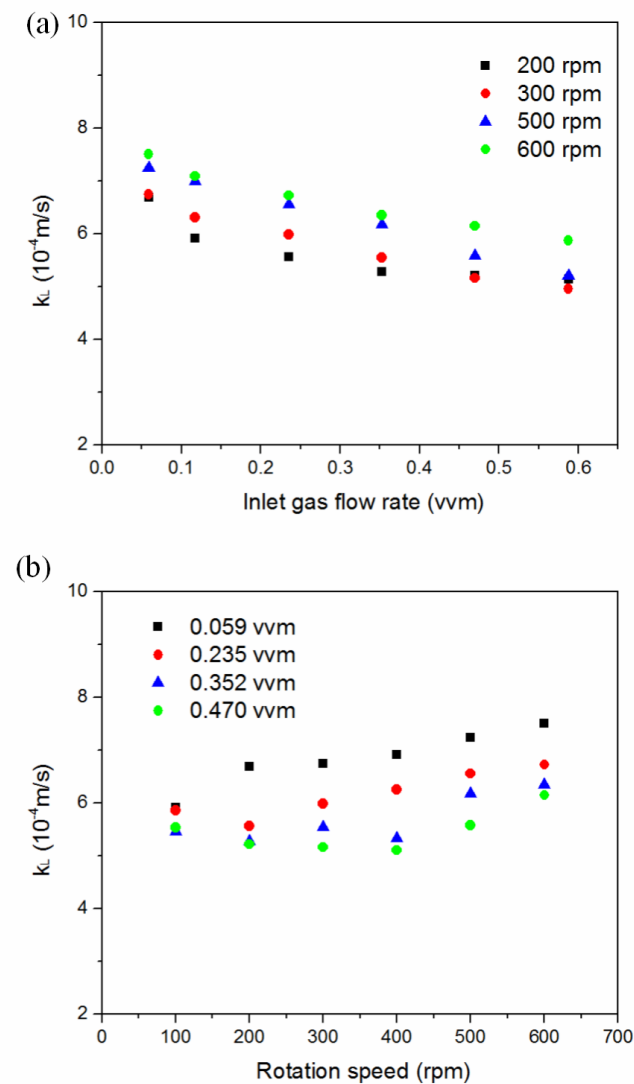

Figure 13: Predicted average mass transfer coefficients by the adaptive mass transfer model under different rotational speeds and inlet gas flow rates.

with gas flow rate, which was also observed in corresponding experimental work [38] and reflected in Eq. 17, is a result of the rapid increase in specific interfacial area $a$ even while $k_{L}$ decreases with increasing gas flow rate. In contrast to the dependence on gas flow rate, $k_{L} a$ is essentially independent of cylinder rotation rate for cylinder speeds below $300 \mathrm{rpm}$, but at higher speeds increases rapidly due to increases in both $k_{L}$ and $a$. This more complicated dependence of $k_{L} a$ on cylinder rotation speed is also mirrored and confirmed by Eq. 17 . 

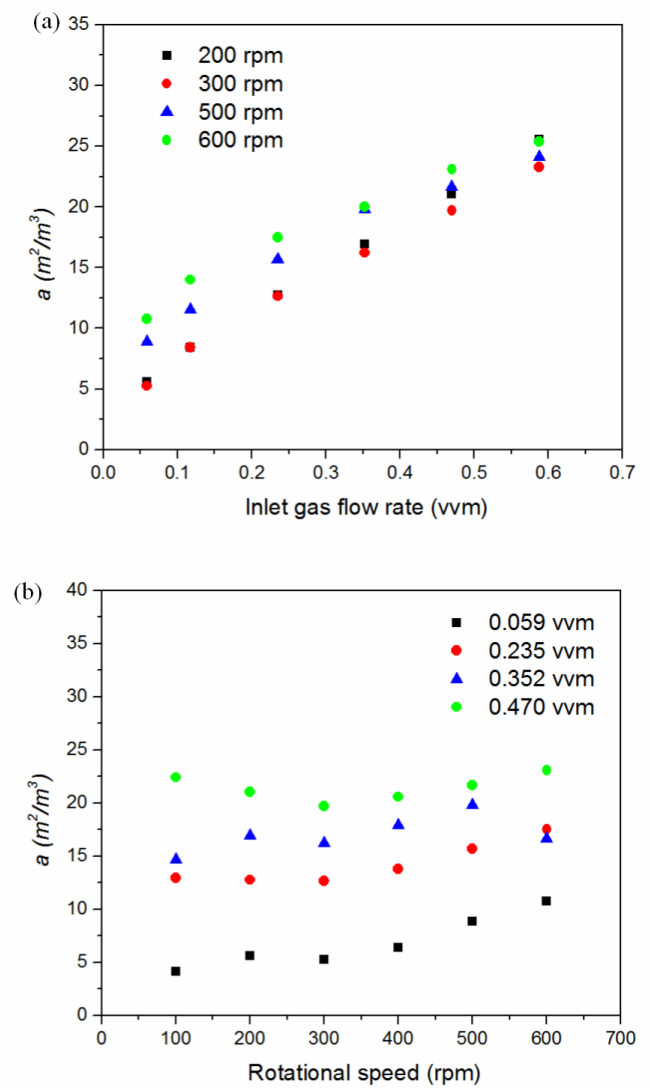

Figure 14: Predicted specific interfacial area as a function of cylinder gas flow rate and cylinder rotation speed.

\section{Conclusions}

Gas-liquid mass transfer in a Taylor vortex reactor was simulated by using a two-fluid CFD model to obtain local phase distribution and velocity field information, which is then used to select an appropriate mass transfer model for computing the volumetric mass transfer coefficient, $k_{L} a$. The two-fluid model, which was previously validated by comparing velocity and phase distribution predictions with experimental measurements in a vertically oriented semibatch gas-liquid Taylor vortex flow device [39], accounts for two-way coupling of sev- 
eral interphase forces including drag, virtual mass, lift, wall lubrication, and turbulent dispersion. The mass transfer model is based upon the Higbie theory, but automatically adapts to the local hydrodynamic environment by computing exposure time using either the penetration theory or eddy cell model based upon the turbulence dissipation rate. This adaptive approach was shown to provide much more accurate predictions for the volumetric mass transfer coefficient than does either model used alone.

After developing the approach described above, the effects of several important operating conditions on local and global mass transfer coefficients were explored. For all flow conditions studied, gas bubbles congregate near the inner cylinder, and therefore most interphase mass transfer also is located near the inner cylinder wall. The cylinder rotation speed, in contrast to the gas feed flow rate, strongly impacts the bubble diameter, slip velocity, and turbulence dissipation rate. The gas flow rate has a greater effect than the cylinder speed on gas holdup, $\alpha_{g}$, but because the holdup is small $(<2 \%)$ even for the highest gas flow rates studied, the rate of interphase mass transfer in general depends more strongly on the azimuthal Reynolds number, in agreement with experimental findings for this system [38].

Further evidence for the validity of the modeling approach described here was obtained by comparing simulation predictions with experimental measurements for the temporal evolution of the concentration of dissolved oxygen at a specific location in the reactor in response to a step change in feed gas composition. A key assumption in determining mass transfer coefficients from the experimental measurements is that the liquid mixing time is short compared to the characteristic mass transfer time. The simulations support this assumption, and consequently the hypothesis that measurements at a single location in the reactor are sufficient for determining the mass transfer coefficient appears to be justified. Lastly, we note that determination of $k_{L} a$ from experimental data at a single location also requires that the local liquid saturation concentration does not depend upon position (gas partial pressure) and only depends upon temperature. Such an assumption can be justified if the gas phase partial pressures do 
not vary strongly with position. In the CFD model presented here, no assumption of spatial homogeneity in the distribution of the gas phase partial pressures is invoked, and the saturation concentration $c_{l, *}$ in Eq. 5 was computed locally. However, because oxygen is in great excess, the value of $c_{l, *}$ is practically constant at all positions in the reactor, consistent with the assumption used to compute $k_{L} a$ from the experimental data. 


\section{Notation}

\begin{tabular}{|c|c|}
\hline$a$ & specific gas-liquid interfacial surface area, $\mathrm{m}^{-1}$ \\
\hline$c_{l}$ & liquid molar concentration, $\mathrm{kmol} \cdot \mathrm{m}^{-3}$ \\
\hline$c_{l *}$ & liquid saturation molar concentration, $\mathrm{kmol} \cdot \mathrm{m}^{-3}$ \\
\hline$C_{D}$ & drag coefficient, dimensionless \\
\hline$d_{b}$ & sauter mean diameter of bubble, $\mathrm{m}$ \\
\hline$D_{L}$ & diffusion coefficient gas in liquid, $\mathrm{m}^{2} \cdot \mathrm{s}^{-1}$ \\
\hline Eo & Eötvös number, dimensionless \\
\hline$H$ & Henry's law constant, $\mathrm{Pa} \cdot \mathrm{m}^{3} \cdot \mathrm{kmol}^{-1}$ \\
\hline$F$ & interphase force, $\mathrm{kg} \cdot \mathrm{m}^{-2} \cdot \mathrm{s}^{-2}$ \\
\hline$g$ & gravitational acceleration, $\mathrm{m} \cdot \mathrm{s}^{-2}$ \\
\hline$G_{k}$ & generation of turbulent kinetic energy, $\mathrm{kg} \cdot \mathrm{m}^{-1} \cdot \mathrm{s}^{-3}$ \\
\hline$G_{\omega}$ & generation of specific dissipation rate, $\mathrm{kg} \cdot \mathrm{m}^{-3} \cdot \mathrm{s}^{-2}$ \\
\hline$k_{l}$ & turbulent kinetic energy of liquid, $\mathrm{m}^{2} \cdot \mathrm{s}^{-2}$ \\
\hline$k_{L}$ & liquid side mass transfer coefficient, $\mathrm{m} \cdot \mathrm{s}^{-1}$ \\
\hline$L$ & cylinder height, m \\
\hline$M_{w}^{i}$ & molecular mass, $\mathrm{kg} \cdot \mathrm{kmol}^{-1}$ \\
\hline$p$ & pressure, $\mathrm{Pa}$ \\
\hline$r$ & cylinder radius, $\mathrm{m}$ \\
\hline$R_{g l}^{i}$ & rate of gas-liquid mass transfer of species $i, \mathrm{kmol} \cdot \mathrm{m}^{-3} \cdot \mathrm{s}^{-1}$ \\
\hline $\mathrm{Re}_{b}$ & bubble Reynolds number, dimensionless \\
\hline $\operatorname{Re}_{a}$ & axial Reynolds number, dimensionless \\
\hline $\operatorname{Re}_{\theta}$ & azimuthal Reynolds number, dimensionless \\
\hline$S$ & net rate of production/destruction, $\mathrm{kg} \cdot \mathrm{m}^{3} \cdot \mathrm{s}$ \\
\hline Sh & Sherwood number, dimensionless \\
\hline$t_{e}$ & exposure time, s \\
\hline$u$ & velocity, $\mathrm{m} \cdot \mathrm{s}^{-1}$ \\
\hline$Y^{i}$ & mass fraction of specie of species $i$, dimensionless \\
\hline$Y_{k}$ & dissipation of turbulent kinetic energy, $\mathrm{kg} \cdot \mathrm{m}^{-1} \cdot \mathrm{s}^{-3}$ \\
\hline$Y_{\omega}$ & dissipation of specific dissipation rate, $\mathrm{kg} \cdot \mathrm{m}^{-3} \cdot \mathrm{s}^{-2}$ \\
\hline
\end{tabular}




\section{Greek letters}

$\begin{array}{ll}\alpha & \text { volume fraction, dimensionless } \\ \varepsilon & \text { turbulence dissipation rate, } \mathrm{m}^{2} \cdot \mathrm{s}^{-3} \\ \omega & \text { specific dissipation rate, } \mathrm{s}^{-1} \\ \nu & \text { kinematic viscosity, } \mathrm{Pa} \cdot \mathrm{s} \\ \mu_{t} & \text { turbulent viscosity, } \mathrm{Pa} \cdot \mathrm{s} \\ \rho & \text { density, } \mathrm{kg} \cdot \mathrm{m}^{-3} \\ \overline{\bar{\tau}} & \text { phase stress tensor, } \mathrm{N} \cdot \mathrm{m}^{-2} \\ \overline{\bar{\tau}} \operatorname{Re} & \text { phase stress tensor, } \mathrm{N} \cdot \mathrm{m}^{-2} \\ \Gamma & \text { aspect ratio, dimensionless } \\ \eta & \text { radius ratio, dimensionless }\end{array}$

\section{Subscripts and Superscripts}

$\begin{array}{ll}b & \text { bubble phase } \\ l & \text { liquid phase } \\ g & \text { gas or global } \\ i & \text { species index }\end{array}$

\section{Acknowledgments}

Financial support was provided for this work by National Science Foundation grant CBET-1236676.

\section{References}

[1] W.-D. Deckwer, R. Burckhart, G. Zoll, Mixing and mass transfer in tall bubble columns, Chemical Engineering Science 29 (11) (1974) 2177-2188.

[2] B. Gourich, C. Vial, N. El Azher, M. B. Soulami, M. Ziyad, Improvement of oxygen mass transfer estimation from oxygen concentration measurements in bubble column reactors, Chemical Engineering Science 61 (18) (2006) $6218-6222$. 
[3] D. Colombet, D. Legendre, A. Cockx, P. Guiraud, F. Risso, C. Daniel, S. Galinat, Experimental study of mass transfer in a dense bubble swarm, Chemical Engineering Science 66 (14) (2011) 3432-3440.

[4] K. Muroyama, K. Imai, Y. Oka, J. Hayashi, Mass transfer properties in a bubble column associated with micro-bubble dispersions, Chemical Engineering Science 100 (2013) 464-473.

[5] Y. Kawase, B. Halard, M. Moo-Young, Theoretical prediction of volumetric mass transfer coefficients in bubble columns for Newtonian and nonNewtonian fluids, Chemical Engineering Science 42 (7) (1987) 1609-1617.

[6] Y. Kawase, N. Hashiguchi, Gas-liquid mass transfer in external-loop airlift columns with newtonian and non-newtonian fluids, The Chemical Engineering Journal and The Biochemical Engineering Journal 62 (1) (1996) $35-42$.

[7] Q. Huang, C. Yang, G. Yu, Z.-S. Mao, CFD simulation of hydrodynamics and mass transfer in an internal airlift loop reactor using a steady two-fluid model, Chemical Engineering Science 65 (20) (2010) 5527-5536.

[8] A. Cockx, Z. Do-Quang, J. Audic, A. Liné, M. Roustan, Global and local mass transfer coefficients in waste water treatment process by computational fluid dynamics, Chemical Engineering and Processing: Process Intensification 40 (2) (2001) 187-194.

[9] F. Garcia-Ochoa, E. Gomez, Theoretical prediction of gas-liquid mass transfer coefficient, specific area and hold-up in sparged stirred tanks, Chemical Engineering Science 59 (12) (2004) 2489-2501.

[10] F. García-Ochoa, E. Gomez, Mass transfer coefficient in stirred tank reactors for xanthan gum solutions, Biochemical Engineering Journal 1 (1) (1998) 1-10. 
[11] F. Garcia-Ochoa, E. Gomez, Prediction of gas-liquid mass transfer coefficient in sparged stirred tank bioreactors, Biotechnology and Bioengineering 92 (6) (2005) 761-772.

[12] B. Özbek, S. Gayik, The studies on the oxygen mass transfer coefficient in a bioreactor, Process Biochemistry 36 (8) (2001) 729-741.

[13] B. Kadam, J. Joshi, S. Koganti, R. Patil, Hydrodynamic and mass transfer characteristics of annular centrifugal extractors, Chemical Engineering Research and Design 86 (3) (2008) 233-244.

[14] S. Wroński, E. Dłuska, R. Hubacz, E. Molga, Mass transfer in gas-liquid Couette-Taylor flow in membrane reactor, Chemical Engineering Science 54 (13) (1999) 2963-2967.

[15] E. Dłuska, S. Wroński, R. Hubacz, Mass transfer in gas-liquid CouetteTaylor flow reactor, Chemical Engineering Science 56 (3) (2001) 1131-1136.

[16] E. Dłuska, S. Wroński, T. Ryszczuk, Interfacial area in gas-liquid CouetteTaylor flow reactor, Experimental Thermal and Fluid Science 28 (5) (2004) 467-472.

[17] B. Haut, H. B. Amor, L. Coulon, A. Jacquet, V. Halloin, Hydrodynamics and mass transfer in a Couette-Taylor bioreactor for the culture of animal cells, Chemical Engineering Science 58 (3) (2003) 777-784.

[18] R. Sorg, T. Tanzeglock, M. Soos, M. Morbidelli, A. Périlleux, T. Solacroup, H. Broly, Minimizing hydrodynamic stress in mammalian cell culture through the lobed Taylor-Couette bioreactor, Biotechnology Journal 6 (12) (2011) 1504-1515.

[19] X. H. Zhu, D. Y. Arifin, B. H. Khoo, J. Hua, C.-H. Wang, Study of cell seeding on porous poly (d, l-lactic-co-glycolic acid) sponge and growth in a Couette-Taylor bioreactor, Chemical Engineering Science 65 (6) (2010) 2108-2117. 
[20] A. M. Kliphuis, L. de Winter, C. Vejrazka, D. E. Martens, M. Janssen, R. H. Wijffels, Photosynthetic efficiency of Chlorella sorokiniana in a turbulently mixed short light-path photobioreactor, Biotechnology Progress 26 (3) (2010) 687-696.

[21] J. Qiao, C. M. J. Lew, A. Karthikeyan, C.-H. Wang, Production of pex protein from qm7 cells cultured in polymer scaffolds in a Taylor-Couette bioreactor, Biochemical Engineering Journal 88 (2014) 179-187.

[22] B. Kong, J. V. Shanks, R. D. Vigil, Enhanced algal growth rate in a Taylor vortex reactor, Biotechnology and Bioengineering 110 (8) (2013) 21402149 .

[23] B. Kong, R. D. Vigil, Light-limited continuous culture of Chlorella vulgaris in a Taylor vortex reactor, Environmental Progress \& Sustainable Energy 32 (4) (2013) 884-890.

[24] B. Kong, R. D. Vigil, Simulation of photosynthetically active radiation distribution in algal photobioreactors using a multidimensional spectral radiation model, Bioresource Technology 158 (2014) 141-148.

[25] C. D. Andereck, S. Liu, H. L. Swinney, Flow regimes in a circular Couette system with independently rotating cylinders, Journal of Fluid Mechanics 164 (1986) 155-183.

[26] D. Coles, Transition in circular Couette flow, Journal of Fluid Mechanics 21 (03) (1965) 385-425.

[27] L. Wang, R. D. Vigil, R. O. Fox, Cfd simulation of shear-induced aggregation and breakage in turbulent Taylor-Couette flow, Journal of Colloid and Interface Science 285 (1) (2005) 167-178.

[28] L. Wang, M. G. Olsen, R. D. Vigil, Reappearance of azimuthal waves in turbulent Taylor-Couette flow at large aspect ratio, Chemical Engineering Science 60 (20) (2005) 5555-5568. 
[29] D. Joseph, K. Nguyen, G. Beavers, Non-uniqueness and stability of the configuration of flow of immiscible fluids with different viscosities, Journal of Fluid Mechanics 141 (1984) 319-345.

[30] Y. Renardy, D. D. Joseph, Couette flow of two fluids between concentric cylinders, Journal of Fluid Mechanics 150 (1985) 381-394.

[31] D. D. Joseph, L. Preziosi, Stability of rigid motions and coating films in bicomponent flows of immiscible liquids, Journal of Fluid Mechanics 185 (1987) 323-351.

[32] X. Zhu, R. J. Campero, R. D. Vigil, Axial mass transport in liquid-liquid Taylor-Couette-Poiseuille flow, Chemical Engineering Science 55 (21) (2000) 5079-5087.

[33] X. Zhu, R. D. Vigil, Banded liquid-liquid Taylor-Couette-Poiseuille flow, AIChE Journal 47 (9) (2001) 1932-1940.

[34] D. P. van Gils, S. G. Huisman, G.-W. Bruggert, C. Sun, D. Lohse, Torque scaling in turbulent Taylor-Couette flow with co-and counterrotating cylinders, Physical Review Letters 106 (2) (2011) 024502.

[35] D. P. van Gils, D. Narezo Guzman, C. Sun, D. Lohse, The importance of bubble deformability for strong drag reduction in bubbly turbulent TaylorCouette flow, Journal of Fluid Mechanics 722 (2013) 317-347.

[36] A. Chouippe, E. Climent, D. Legendre, C. Gabillet, Numerical simulation of bubble dispersion in turbulent Taylor-Couette flow, Physics of Fluids (1994-present) 26 (4) (2014) 043304.

[37] R. Maryami, S. Farahat, M. S. Mayam, et al., Bubbly drag reduction in a vertical Couette-Taylor system with superimposed axial flow, Fluid Dynamics Research 46 (5) (2014) 055504.

[38] M. Ramezani, B. Kong, X. Gao, M. G. Olsen, R. D. Vigil, Experimental measurement of oxygen mass transfer and bubble size distribution in an airwater multiphase Taylor-Couette vortex bioreactor, Chemical Engineering 
Journal 279 (2015) 286 - 296. doi:http://dx.doi.org/10.1016/j.cej . 2015.05 .007 .

[39] X. Gao, B. Kong, R. D. Vigil, CFD investigation of bubble effects on Taylor-Couette flow patterns in the weakly turbulent vortex regime, Chemical Engineering Journal 270 (2015) 508 - 518. doi:http://dx.doi.org/ $10.1016 / j$.cej.2015.02.061.

[40] X. Gao, B. Kong, R. D. Vigil, CFD investigation of bubbly turbulent Taylor-Couette flow, Chinese Journal of Chemical Engineering (submitted) $\mathrm{xx}(\mathrm{x})(2015) \mathrm{xxxxx}$.

[41] M. J. Sathe, S. S. Deshmukh, J. B. Joshi, S. B. Koganti, Computational fluid dynamics simulation and experimental investigation: Study of twophase liquid- liquid flow in a vertical taylor- couette contactor, Industrial \& Engineering Chemistry Research 49 (1) (2009) 14-28.

[42] X. Gao, L.-J. Wang, C. Wu, Y.-W. Cheng, X. Li, Novel bubble-emulsion hydrodynamic model for gas-solid bubbling fluidized beds, Industrial \& Engineering Chemistry Research 52 (31) (2013) 10835-10844.

[43] S. M. Monahan, R. O. Fox, Effect of model formulation on flow-regime predictions for bubble columns, AICHE Journal 53 (1) (2007) 9-18. doi: \{10.1002/aic.11042\}.

[44] A. Buffo, D. L. Marchisio, Modeling and simulation of turbulent polydisperse gas-liquid systems via the generalized population balance equation, Reviews in Chemical Engineering 30 (1) (2014) 73-126. doi:\{10.1515/ revce-2013-0015\}.

[45] A. Tomiyama, I. Kataoka, I. Zun, T. Sakaguchi, Drag coefficients of single bubbles under normal and micro gravity conditions., JSME International Journal Series B 41 (2) (1998) 472-479.

[46] D. Drew, R. Lahey, Analytical modeling of multiphase flow, Particulate Two-Phase Flow (1993) 509-566. 
[47] S. Antal, R. Lahey, J. Flaherty, Analysis of phase distribution in fully developed laminar bubbly two-phase flow, International Journal of Multiphase Flow 17 (5) (1991) 635-652.

[48] S. Talvy, A. Cockx, A. Liné, Modeling hydrodynamics of gas-liquid airlift reactor, AIChE Journal 53 (2) (2007) 335-353.

[49] A. Behzadi, R. Issa, H. Rusche, Modelling of dispersed bubble and droplet flow at high phase fractions, Chemical Engineering Science 59 (4) (2004) 759-770.

[50] Y. Sato, K. Sekoguchi, Liquid velocity distribution in two-phase bubble flow, International Journal of Multiphase Flow 2 (1) (1975) 79-95.

[51] D. C. Wilcox, et al., Turbulence modeling for CFD, Vol. 2, DCW industries La Canada, CA, 1998.

[52] R. Higbie, The rate of absorption of a pure gas into still liquid during short periods of exposure, Trans. Am. Inst. Chem. Eng. 31 (1935) 365.

[53] J. C. Lamont, D. Scott, An eddy cell model of mass transfer into the surface of a turbulent liquid, AIChE Journal 16 (4) (1970) 513-519.

[54] R. Griffith, Mass transfer from drops and bubbles, Chemical Engineering Science 12 (3) (1960) 198-213.

[55] A. Lochiel, P. Calderbank, Mass transfer in the continuous phase around axisymmetric bodies of revolution, Chemical Engineering Science 19 (7) (1964) 471-484.

[56] V. Linek, M. Kordač, M. Fujasová, T. Moucha, Gas-liquid mass transfer coefficient in stirred tanks interpreted through models of idealized eddy structure of turbulence in the bubble vicinity, Chemical Engineering and Processing: Process Intensification 43 (12) (2004) 1511-1517. 
[57] S. S. Alves, J. M. Vasconcelos, S. P. Orvalho, Mass transfer to clean bubbles at low turbulent energy dissipation, Chemical Engineering Science 61 (4) (2006) 1334-1337.

[58] T. Wang, J. Wang, Numerical simulations of gas-liquid mass transfer in bubble columns with a CFD-PBM coupled model, Chemical Engineering Science 62 (24) (2007) 7107-7118.

[59] S. Patankar, Numerical Heat Transfer and Fluid Flow, CRC Press, 1980.

[60] Y. Murai, H. Oiwa, Y. Takeda, Bubble behavior in a vertical TaylorCouette flow, in: Journal of Physics: Conference Series, Vol. 14, IOP Publishing, 2005, p. 143.

[61] M. Simonnet, C. Gentric, E. Olmos, N. Midoux, Experimental determination of the drag coefficient in a swarm of bubbles, Chemical Engineering Science 62 (3) (2007) 858-866. doi: $\{10.1016 / j$.ces .2006.10.012\}.

[62] I. Roghair, M. V. S. Annaland, H. J. A. M. Kuipers, Drag Force and Clustering in Bubble Swarms, AICHE Journal 59 (5) (2013) 1791-1800. doi:\{10.1002/aic.13949\}. 